\title{
Heterologous transporter expression for improved fatty alcohol secretion in yeast
}

\author{
Yating $\mathrm{Hu}^{\mathrm{a}}$, Zhiwei Zhu ${ }^{\mathrm{a}, \mathrm{b}}$, Jens Nielsen ${ }^{\mathrm{a}, \mathrm{b}, \mathrm{c}, \mathrm{d}}$, Verena Siewers ${ }^{\mathrm{a}, \mathrm{b}, *}$ \\ a Department of Biology and Biological Engineering, Chalmers University of Technology, SE-41296 Gothenburg, Sweden \\ b Novo Nordisk Foundation Center for Biosustainability, Chalmers University of Technology, SE-41296 Gothenburg, Sweden \\ ${ }^{\mathrm{c}}$ Novo Nordisk Foundation Center for Biosustainability, Technical University of Denmark, DK-2800 Kgs. Lyngby, Denmark \\ d Science for Life Laboratory, Royal Institute of Technology, SE-17121 Stockholm, Sweden
}

\section{A R T I C L E I N F O}

\section{Keywords:}

Fatty alcohol transporter

Secretion

S. cerevisiae

Tolerance

\begin{abstract}
A B S T R A C T
The yeast Saccharomyces cerevisiae is an attractive host for industrial scale production of biofuels including fatty alcohols due to its robustness and tolerance towards harsh fermentation conditions. Many metabolic engineering strategies have been applied to generate high fatty alcohol production strains. However, impaired growth caused by fatty alcohol accumulation and high cost of extraction are factors limiting large-scale production. Here, we demonstrate that the use of heterologous transporters is a promising strategy to increase fatty alcohol production. Among several plant and mammalian transporters tested, human FATP1 was shown to mediate fatty alcohol export in a high fatty alcohol production yeast strain. An approximately five-fold increase of fatty alcohol secretion was achieved. The results indicate that the overall cell fitness benefited from fatty alcohol secretion and that the acyl-CoA synthase activity of FATP1 contributed to increased cell growth as well. This is the first study that enabled an increased cell fitness for fatty alcohol production by heterologous transporter expression in yeast, and this investigation indicates a new potential function of FATP1, which has been known as a free fatty acid importer to date. We furthermore successfully identified the functional domain of FATP1 involved in fatty alcohol export through domain exchange between FATP1 and another transporter, FATP4. This study may facilitate a successful commercialization of fatty alcohol production in yeast and inspire the design of novel cell factories.
\end{abstract}

\section{Introduction}

Owing to the high global demand of petroleum, and concerns about energy security and climate change, alternative transportation fuels produced by microbes have drawn much attention as sustainable and renewable replacements for petroleum-based fuels (Kerr, 2007; Stephanopoulos, 2007). To meet the increasing needs of energy, many efforts on metabolic engineering of microbes have been made to produce chemicals that can be used as drop-in fuels, such as fatty acids (Rutter et al., 2015), fatty alcohols (Tang and Chen, 2015; Youngquist et al., 2013) and alkanes (Rutter et al., 2015). However, the toxicity of these chemicals is a major challenge for scaling up production. Highyield production requires stable and robust host cells that are tolerant against these toxic chemicals. Increasing the tolerance seems to be a promising strategy to improve the yields, and several studies succeeded in overcoming biofuel toxicity by different engineering strategies. For instance, global transcription machinery engineering (gTME) was used to improve ethanol tolerance in Saccharomyces cerevisiae (Alper et al.,
2006). In another study, the tolerance to isobutanol in Escherichia coli was increased by adaptive evolution to benefit isobutanol production (Minty et al., 2011).

A different approach is transporter engineering. For instance, sugar utilization for biofuel production was improved by engineering xylose/ glucose importers (Hamacher et al., 2002; N. Li et al., 2016; Nijland et al., 2016; Shin et al., 2017). Likewise, the yields of many biofuel molecules (butanol, isopentanol and limonene, etc.) were increased by exporter engineering, which improved cell survival (Dunlop et al., 2011). For instance, the tolerance to short-chain alcohols was enhanced in E. coli by engineering the AcrB efflux pump, and Turner and Dunlop improved biofuel tolerance by combining two types of efflux pumps in E. coli (Fisher et al., 2014; Turner and Dunlop, 2015).

Efflux pumps contribute to the export of a wide range of chemicals, and by this, the viability of cells can be significantly increased. In many cases, transporters appear to be specific for certain compounds or compound classes, for example, SrpABC plays a key role in tolerance of Pseudomonas putida S12 to hexane, octanol and other hydrocarbons

\footnotetext{
* Corresponding author at: Department of Biology and Biological Engineering, Chalmers University of Technology, SE-41296 Gothenburg, Sweden.

E-mail address: siewers@chalmers.se (V. Siewers).
} 
(Dunlop, 2011; Kieboom et al., 1998). Export of biofuel compounds with transporters from the resistance-nodulation-division (RND) family and ATP-binding cassette (ABC) family in Gram-negative bacteria offers a promising strategy for increasing biofuel production and tolerance (Doshi et al., 2013; Minty et al., 2011). However, for Saccharomyces cerevisiae, which is more suitable as an industrial-scale production host, reports concerning the use of transporters for improved biofuel secretion and production are scarce. In addition, for some high economically valuable biofuels such as fatty alcohols, substrate-specific transporters have not been identified yet.

Fatty alcohols, which can be derived from fatty acids, are currently widely used as detergents, skin care products, cosmetics, medicines, and also serve as biodiesel candidates (Fortman et al., 2008; Zhou et al., 2014). Although metabolic engineering strategies have been pursued to achieve high production of fatty alcohols in $S$. cerevisiae, major obstacles for industrial production are the slow growth of the producing cells and high costs for extraction due to the fact that most of the fatty alcohols are stored inside the cells (Zhou et al., 2016). Here, we enhanced fatty alcohol secretion by expressing the fatty acid transporter FATP1 from human in yeast (Martin et al., 2000). A new function of FATP1 was observed, as this shows that FATP1 is not only able to mediate fatty acid uptake (Hirsch et al., 1998; Schaffer and Lodish, 1994), but can also facilitate fatty alcohol export in yeast. Through expression of chimetic forms of FATP1 with segments from a close homolog FATP4, which does not have fatty alcohol exporting activity, we furthermore identified the protein domain involved in fatty alcohol transport.

\section{Materials and methods}

\subsection{Strains and plasmids}

The $S$. cerevisiae strain FOH33 [MAT $\alpha$ MAL2-8 ${ }^{c}$ SUC2 his3A ura3-52

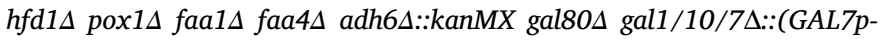
MmCAR-ADH1t) + (GAL3p-npgA-FBA1t); pAOH9] constructed as previously published (Zhou et al., 2016), but with an additional deletion in HIS3 was used as fatty alcohol production strain. The plasmids constructed in this study were all introduced into this host strain.

HXT7p and HXT6p promoters were amplified from the yeast genome using primers 6-HXT7-F/ HXT7-R-BamHI and NotI-HXT6-F/ HXT6-R-7, respectively. After fusing them together by PCR, the promoter cassette and pIYC04 (Chen et al., 2013) were digested by NotI and BamHI, then the PGK1p-TEF1p fragment from pIYC04 was replaced by the HXT7pHXT6p promoter cassette to form pIYH01 as backbone plasmid in this study. All the yeast strains and plasmids are listed in Table S1. The primers are listed in Table S2. All the codon optimized heterologous genes were synthesized (Genscript, Piscataway, NJ, USA) and are listed in Table S3.

\subsection{Construction of expression plasmids}

All heterologous genes were amplified using corresponding primers, which contained sequences overlapping with backbone plasmid pIYH01. pIYH01 was divided into two fragments, which were amplified by two pairs of primers, 01-HXT6-F/ 01-amp-R and 01-amp-F/ 01-R, respectively. Fragments from genes and vector generated new expression plasmids by Gibson assembly cloning (Gibson et al., 2009). All of the heterologous transporter genes were under the control of HXT6p except ABCG11. ABCG11 was introduced under the control of HXT7p, and expressed together with $A B C G 9, A B C G 12$ and $A B C G 14$, respectively, to result in formation of heterodimers in yeast.

Three different segments from both FATP1 and FATP4 were amplified by the primers listed in Table S1: F1- the first part of FATP1 (corresponding to amino acid residues 1-246), F2- the second part of FATP1 (amino acid residues 247-503), F3- the third part of FATP1 (amino acid residues 504-646), F4- the first part of FATP4 (amino acid residues 1-243), F5- the second part of FATP4 (amino acid residues 244-500), and F6- the third part of FATP4 (amino acid residues 501-643). Three of those segments were fused with each other to generate six new FATP isoforms shown in Fig. 3a. The plasmid pIYH01 as the backbone was digested by restriction enzymes PacI and NotI. All the new FATP genes were constructed and integrated into the backbone plasmid by Gibson assembly cloning.

To fuse the GFP with FATP1, the backbone plasmid pIYH01 was digested by NotI and SacI. GFP was fused with the C-terminus of FATP1 introducing a flexible linker, GGGGS. FATP1 and GFP were amplified using primers FATP1-notI-F/ FATP1-GFP-R and GGGGS-GFP-F/GFPsacI-R, respectively. We fused these two fragments by PCR, and the fused fragment was digested by NotI and SacI. Then the FATP1-GFP fragment was joined with the backbone plasmid, resulting in pIYH25.

To introduce the point mutations into FATP1, pIYH01 was digested by NotI and SgsI as a backbone fragment. Then, an 800 bp fragment was amplified from pIYH02 using primers SgsI-FATP1-F/ Ala-FATP1-R. Hereby, the mutant $\mathrm{Asp}^{492} \mathrm{Ala}$ codon was introduced into this fragment, and this fragment was used as a primer together with another primer, NotI-FATP1-R, to amplify FATP1 from pIYH02. The 2300 bp fragment containing FATP (Asp ${ }^{492}$ Ala) was digested by SgsI and NotI and then ligated with the backbone fragment resulting in pIYH23. The same method was used to form pIYH24, which contained FATP (Asp ${ }^{492} \mathrm{Glu}$ ).

\subsection{Media and culture conditions}

SD-Ura medium was used as pre-culture medium for yeast transformation and contained $6.7 \mathrm{~g} / \mathrm{L}$ yeast nitrogen base (YNB) without amino acids (Formedium, Hunstanton, UK), $0.77 \mathrm{~g} / \mathrm{L}$ Complete Supplement Mixture (CSM) w/o uracil (Formedium), and $20 \mathrm{~g} / \mathrm{L}$ glucose (Merck, Darmstadt, Germany). Strains containing URA3-based and HIS3-based plasmids were selected on synthetic complete medium without uracil and histidine (SD-Ura-His), which consisted of $6.7 \mathrm{~g} / \mathrm{L}$ yeast nitrogen base without amino acid (Formedium), $0.77 \mathrm{~g} / \mathrm{L} \mathrm{CSM} \mathrm{w/}$ o uracil-histidine (Formedium), $20 \mathrm{~g} / \mathrm{L}$ glucose and $18 \mathrm{~g} / \mathrm{L}$ agar. For shake flask fermentations, the minimal medium contained $7.5 \mathrm{~g} / \mathrm{L}$ $\left(\mathrm{NH}_{4}\right)_{2} \mathrm{SO}_{4}, 14.4 \mathrm{~g} / \mathrm{L} \mathrm{KH} \mathrm{KO}_{4}, 0.5 \mathrm{~g} / \mathrm{L} \mathrm{MgSO}_{4} \cdot 7 \mathrm{H}_{2} \mathrm{O}, 20 \mathrm{~g} / \mathrm{L}$ glucose, $2 \mathrm{~mL} / \mathrm{L}$ trace metal solution, $1 \mathrm{~mL} / \mathrm{L}$ vitamin solution (Verduyn et al., 1992). The $\mathrm{pH}$ of the medium was adjusted to 6.0 by addition of $2 \mathrm{M}$ $\mathrm{KOH}$. Twenty milliliter of minimal medium in $100 \mathrm{~mL}$ unbaffled cottonstopped flasks were inoculated with an amount of the respective yeast strain to reach a starting OD600 of 0.1. All the yeast strains were grown at $30{ }^{\circ} \mathrm{C}$ and $200 \mathrm{rpm}$ for $72 \mathrm{~h}$.

\subsection{Analytical measurements}

Samples were taken to measure the biomass and concentration of fatty alcohols after $72 \mathrm{~h}$. The dry cell weight (DCW) was determined by filtering $5 \mathrm{~mL}$ of broth through a weighed $0.45 \mu \mathrm{m}$ filter membrane (Sartorius AG, Göttingen, Germany) and measuring the increased weight of the dry filter. Extracellular fatty acids were extracted and methylated by dichloromethane containing methyl iodide as methyl donor. About $100 \mu \mathrm{L}$ of cell cultures from shake flasks after $72 \mathrm{~h}$ were diluted with $200 \mu \mathrm{L}$ water. All of the dilutions were transferred into glass extraction vials, then $10 \mu \mathrm{L} 40 \%$ tetrabutylammonium hydroxide was added immediately followed by addition of $200 \mu \mathrm{L}$ dichloromethane which contained $200 \mathrm{mM}$ methyl iodide and $100 \mathrm{mg} / \mathrm{L}$ pentadecanoic acid as an internal standard. After shaking for $30 \mathrm{~min}$ at $1400 \mathrm{rpm}$, the mixtures were centrifuged for $10 \mathrm{~min}$ at $5000 \mathrm{~g}$ to separate organic layer and aqueous layer. A $150 \mu \mathrm{L}$ dichloromethane layer was transferred into a new vial, and evaporated until dryness at room temperature. Then, $150 \mu \mathrm{L}$ hexane was added to the vial to resuspend the extracted methyl esters. The extracted FAMEs were analyzed by gas chromatography (Focus GC, ThermoFisher Scientific, Waltham, MA, USA) with a Zebron ZB-5MS GUARDIAN capillary column $(30 \mathrm{~m} \times$ $0.25 \mathrm{~mm} \times 0.25 \mu \mathrm{m}$, Phenomenex, Torrance, CA, USA) and a DSQII 
mass spectrometer (ThermoFisher Scientific). The program was as follow: The initial temperature of $40{ }^{\circ} \mathrm{C}$ was held for $2 \mathrm{~min}$, then ramped to $130{ }^{\circ} \mathrm{C}$ at a rate of $30{ }^{\circ} \mathrm{C}$ per minute, then raise to $280{ }^{\circ} \mathrm{C}$ at a rate of $10^{\circ} \mathrm{C}$ per min and held for $3 \mathrm{~min}$. The temperatures of inlet, mass transfer line and ion source were kept at 280,300 and $230{ }^{\circ} \mathrm{C}$, respectively (Zhou et al., 2016). The injection volume was $1 \mu \mathrm{L}$. The flow rate of the carrier gas (helium) was set to $1.0 \mathrm{~mL} / \mathrm{min}$, and data were acquired at full-scan mode $(50-650 \mathrm{~m} / \mathrm{z})$.

For quantification of intracellular fatty alcohols, cell pellets were collected by centrifugation from $2 \mathrm{~mL}$ aliquots of cell culture, then the cells were subjected to freeze drying for $48 \mathrm{~h}$. Fatty alcohols were extracted by $4 \mathrm{~mL}$ of chloroform-methanol $(2: 1, \mathrm{v} / \mathrm{v})$ which contained pentadecanol $(0.01 \mathrm{mg} / \mathrm{mL})$ as internal standard. Samples were vigorously vortexed and then placed in the microwave reaction vessel $(12 \mathrm{~cm}$ $\times 3 \mathrm{~cm}$ I.D., $0.5 \mathrm{~cm}$ thickness; Milestone Start D, Sorisole, Italy). The thermal program of the microwave was ramped to $60{ }^{\circ} \mathrm{C}$ within $6 \mathrm{~min}$ from room temperature and kept for 10 min (Khoomrung et al., 2013). After the samples had cooled down to room temperature, $1 \mathrm{~mL} \mathrm{NaCl}$ $(0.73 \% \mathrm{w} / \mathrm{v})$ was added. The organic phase was transferred into a new glass tube after centrifuging at $1000 \times g$ for $10 \mathrm{~min}$. Samples were then concentrated by drying under vacuum, and re-suspended with $200 \mu \mathrm{L}$ ethyl acetate. The same equipment of gas chromatography as described above was used for fatty alcohol analysis. The GC program was as follows: The initial temperature of $45^{\circ} \mathrm{C}$ was held for $2.5 \mathrm{~min}$, then ramped to $220^{\circ} \mathrm{C}$ at a rate of $20^{\circ} \mathrm{C}$ per min and held for 2 min before ramping to $300{ }^{\circ} \mathrm{C}$ at a rate of $20^{\circ} \mathrm{C}$ per min and being held for 5 min. The temperatures of inlet, mass transfer line and ion source were kept at 250, 300 and $230{ }^{\circ} \mathrm{C}$, respectively. The flow rate of the carrier gas (helium) was set at $1.0 \mathrm{~mL} / \mathrm{min}$, and data were acquired at full-scan mode $(50-650 \mathrm{~m} / \mathrm{z})$.

For extraction of extracellular fatty alcohols, $2 \mathrm{~mL}$ aliquots of supernatant were collected from cell cultures after centrifuging for $10 \mathrm{~min}$ at $2000 \mathrm{~g}$. Fatty alcohols were extracted by adding $1 \mathrm{~mL}$ ethyl acetate with pentadecanol as internal standard. The samples were then vortexed at $1400 \mathrm{rpm}$ for $30 \mathrm{~min}$ and after phase separation by centrifugation, the organic phase was transferred into a new GC vial. The same analysis GC program as described above was used. Final quantification of all samples were performed by the Xcalibur software.

\subsection{Fluorescence microscopy analysis}

Plasmid pIYH25 (see above) was used to transform the yeast strain FOH33. The cells were cultivated in minimal medium for $30 \mathrm{~h}$ at $30^{\circ} \mathrm{C}$, $200 \mathrm{rpm}$. Three $\mu \mathrm{L}$ of the cell culture were dropped on a microscope slide and viewed by a LEICA DMI4000 microscope (Leica Microsystems GmbH, Wetzlar, Germany) with GFP and DIC filters.

\section{Results}

\subsection{Transporter screening}

In a previous study, we rewired yeast metabolism for fatty alcohol production (Zhou et al., 2016). Based on a high free fatty acid producing strain, enzymes involved in fatty alcohol synthesis had been (over) expressed, including the heterologous reductases MmCAR and FaCoAR as well as endogenous alcohol dehydrogenase Adh5. In addition, the genes POX1, FAA1, FAA4, HFD1, and ADH6 responsible for competing reactions had been deleted in the same strain. The final strain, FOH33, produced around $1.5 \mathrm{~g} / \mathrm{L}$ fatty alcohols in fed-batch cultivations, which however led to impaired growth, probably due to toxicity of intracellularly accumulated fatty alcohols. In order to relieve the cells from product toxicity, we attempted to identify transporters that facilitate fatty alcohol export in this strain. Substrate specificity of known transporters was the theoretical basis for choosing transporter candidates. Since fatty acids and fatty alcohols have similar structures, we assumed that transporters for fatty acids and related lipids could also recognize fatty alcohols as substrates. Several fatty acid and wax transporters from human and plants were therefore selected and expressed in S. cerevisiae FOH33 (strains YH02-10 in Supplementary Table S1). FATP1, FATP4, CD36, and ABCB1 are transporters involved in fatty acid uptake in human cells, while ABCG9, ABCG11, ABCG12, and ABCG14, which function as homodimers or heterodimers, are membrane proteins from the G subfamily of $\mathrm{ABC}$ transporters in Arabidopsis thaliana (Table 1).

The engineered strains were grown in shake flasks and extracellular fatty alcohols were extracted and quantified. Among all these transporters evaluated, only FATP1 dramatically increased the final titer of extracellular fatty alcohols, i.e. around $70 \mathrm{mg} / \mathrm{L}$ compared to $16 \mathrm{mg} / \mathrm{L}$ produced by the control strain (Fig. S1). Interestingly, FATP1 also improved the growth of the engineered yeast strain, and resulted in an $83 \%$ increase of final cell mass (Fig. S1), suggesting that expression of FATP1 relieved the toxicity of intercellular fatty alcohols by exporting them out of the cells. For almost all of the other transporters, the final OD600 of the strains was even reduced compared to the control. Furthermore, the final concentrations of extracellular fatty alcohols were lower than found for the control strain.

FATP1 belongs to the FATP family with six FATP isoforms in human, referred to as FATP1-6, which are fatty acid transporters expressed in many tissues, including liver, brain, lung, heart, skin, etc (Chiu et al., 2005; Gimeno et al., 2003; Herrmann et al., 2001a; Hirsch et al., 1998; Schmuth et al., 2005). All members are characterized by the presence of an FATP signature sequence, a well-conserved 311amino acid sequence (Stahl, 2001). They have previously been identified as dual functional proteins, which mediate fatty acid uptake and show fatty acyl-CoA synthetase activity in human cells (Coe et al., 1999; Herrmann et al., 2001b; Hirsch et al., 1998; Mihalik et al., 2002). It had been shown that all of these isoforms located to the plasma membrane when expressed in yeast, but each of them showed different capacities in fatty acid uptake and activation (DiRusso et al., 2005). Several studies indicated that the different isoforms activated specific fatty acids with different efficiencies (DiRusso et al., 2005; Pei et al., 2004; Stahl, 2001). Based on previous studies, FATP1 is known as a fatty acid transport protein in human cells, where it enhances the uptake of longchain fatty acids (LCFA) (Hirsch et al., 1998; Schaffer and Lodish, 1994). Similar properties - facilitation of long-chain fatty acid uptake were observed when a yeast strain expressing FATP1 grew on longchain fatty acids as the sole carbon source (DiRusso et al., 2005).

In order to investigate if other FATP isoforms also promote extracellular fatty alcohol production, all six isoforms of the FATP family, FATP1-6, were expressed in the FOH33 strain. Surprisingly, none of the other isoforms facilitated fatty alcohol export as FATP1, and less extracellular fatty alcohols were detected by GC-MS (Fig. 1A). In addition, there was no positive effect on the cell growth for FATP2, FATP3, FATP5, and FATP6 expressing strains, except for the strain expressing FATP4, for which the final OD600 was around 9, the same as for the FATP1 expressing strain (Fig. 1B). Although expression of FATP4 also improved cell growth of the FOH33 strain, it did not show any fatty alcohol export activity. This result indicated that the fatty alcohol export function was specific to FATP1.

\subsection{Analysis of FATP1 expressing strain}

When FATP1 was expressed in FOH33, around $70 \mathrm{mg} / \mathrm{L}$ extracellular fatty alcohols were obtained from shake-flask cultivations, which was 4.5-fold higher than the final titer of the control strain (ca. $15 \mathrm{mg} / \mathrm{L}$ ) (Fig. 2A). The titer of intracellular fatty alcohols increased by $40 \%$ in the FATP1 strain, that is $170 \mathrm{mg} / \mathrm{L}$ compared to $120 \mathrm{mg} / \mathrm{L}$ in the control strain (Fig. 2B). The titer of extracellular fatty acids was $190 \mathrm{mg} / \mathrm{L}$, demonstrating that the level of extracellular fatty acids was also enhanced compared with the control strain (60 mg/L) (Fig. 2C). In additional, we fused FATP1 with GFP to verify the expression and localization using a fluorescence microscope. It can be seen that FATP1 


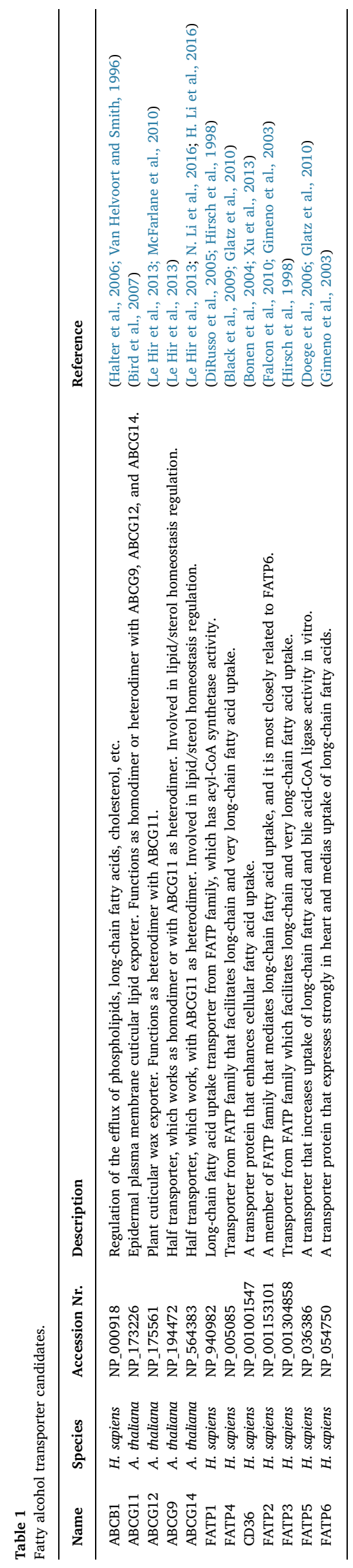

was well expressed in FOH33 yeast strain, and located at least partially to the plasma membrane (Fig. S2). As mentioned above, we found that FATP1 expression was beneficial for cell growth as it enabled the FOH33 strain to achieve a faster growth rate and higher final cell mass than the control strain (Fig. 2D).

In order to determine whether the increased extracellular fatty alcohols resulted from the higher biomass concentration, the specific yield coefficient of fatty alcohols needs to be considered. The results showed that the specific yield of total fatty alcohols (both intra- and extracellular fraction) was not dramatically changed and in fact slightly lower in the FATP1 strain in comparison to the control strain (Fig. 2E). However, the distribution of extracellular and intracellular fatty alcohols based on yields showed a clear difference in these two strains. The profiles of extracellular and intracellular fatty alcohols demonstrated that the yield of extracellular fatty alcohols from the FATP1 strain was $6.4 \mathrm{mg} / \mathrm{L} / \mathrm{OD}$, compared to only $2.4 \mathrm{mg} / \mathrm{L} / \mathrm{OD}$ for the control strain, an about 2.6-fold increase (Fig. $2 E$ ). The results also showed that the yield of intracellular fatty alcohols $(16.1 \mathrm{mg} / \mathrm{L} / \mathrm{OD}$ compared to $25.4 \mathrm{mg} / \mathrm{L} /$ OD in the control strain) decreased significantly after FATP1 expression, which helped the cells to cope with the alcohol toxicity. In conclusion, FATP1 expression benefitted fatty alcohol secretion. The level of extracellular fatty alcohols and their total (intracellular and extracellular) titer increased about 4.5- and 2-fold, respectively. Furthermore, FATP1 expression led to an improved cell growth, and a more than 2-fold increase in biomass was achieved compared to the control strain.

\subsection{Identification of protein domain involved in fatty alcohol secretion}

From the results in this study, we concluded that only one FATP isoform (FATP1) facilitated fatty alcohol export. Still, FATP4 was able to improve growth of $\mathrm{FOH} 33$, which might potentially be due to its function as acyl-CoA synthetase. We analyzed the intracellular fatty alcohols of FATP4 expressing strain in a new experiment. The results showed that the FATP4 expressing strain $(112.7 \mathrm{mg} / \mathrm{L})$ has a similar titer of intracellular fatty alcohols as the parental strain $(109.2 \mathrm{mg} / \mathrm{L})$ and a lower titer than the FATP1 expressing strain (155.4 mg/L) (Fig. $\mathrm{S} 3 A$ ). Accordingly, the intracellular yield was the highest in the control (18.95 mg/L/OD), lower in the FATP1 expressing strain $(15.53 \mathrm{mg} / \mathrm{L} /$ OD) and even lower in the FATP4 expressing strain (12.49 mg/L/OD). This indicated that the fatty acid activating activity of FATP4 might compete with fatty alcohol biosynthesis, which led to reduced intracellular fatty alcohol levels and thus improved cell growth (Fig. S3B). Therefore, the results also showed that there might be no direct connection between fatty alcohol export and fatty acid activation activities. The fatty acid import mediated by murine FATP4 had been shown to be more efficient than that of FATP1 (DiRusso et al., 2008). In our study, FATP1 was found to be the only heterologous protein among the tested ones, which benefits fatty alcohol secretion in yeast. The human transporters of the FATP family contain an intrinsic well conserved FATP/VLCS motif, and an ATP/AMP motif, which contributes to fatty acyl-CoA synthetase activity and is commonly found in all adenylateforming enzymes (Black et al., 2000, 1997; Watkins et al., 1999; Zou et al., 2002). These amino acid sequence elements serve to distinguish FATPs from sole fatty acyl-CoA synthetases (FACS) and fatty acid transporters.

To determine which protein domain of FATP1 can confer the ability to transport fatty alcohols to FATP4, a domain exchange strategy was applied between FATP1 and FATP4. Each gene was divided into three domains based on the corresponding amino acid sequence. The $\mathrm{N}$ terminal parts (F1 and $\mathrm{F} 4)$ were the regions upstream to the respective ATP/AMP motifs of both FATP transporters, the middle domains (F2 and F5) contained the ATP/AMP motif and covered 257 amino acids in total, and the last 143 amino acids formed the C-terminal domains (F3 and F6) with the FATP/VLCS motif (Fig. 3A). The three domains from FATP1 and FATP4, respectively, were fused with each other to generate 
A

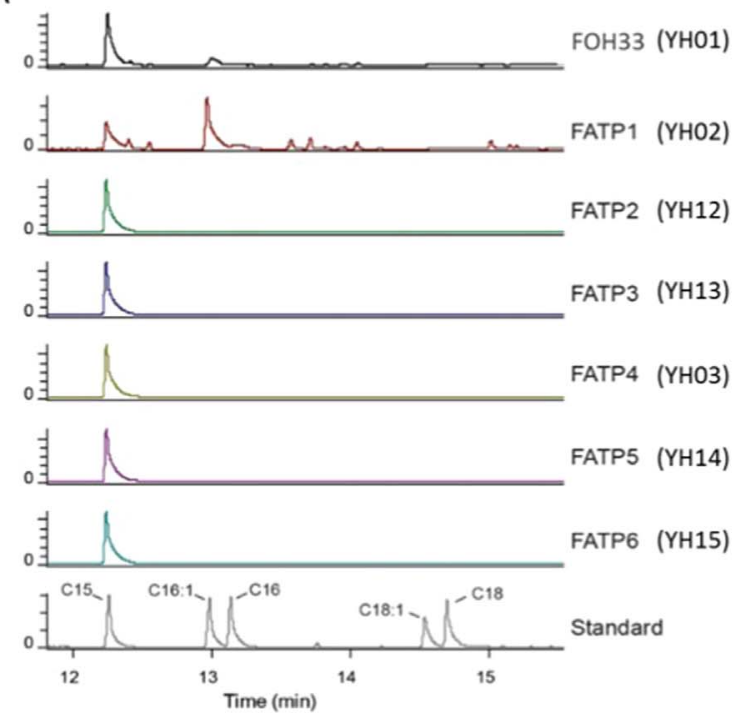

B

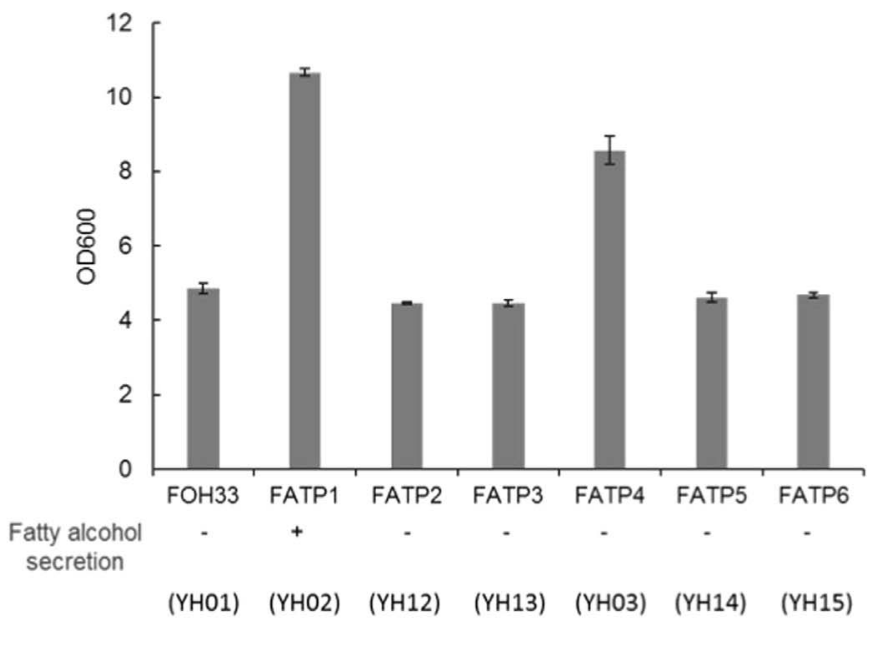

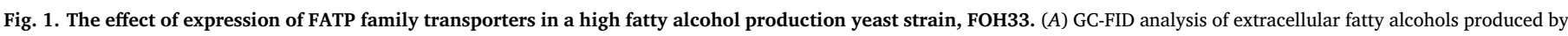

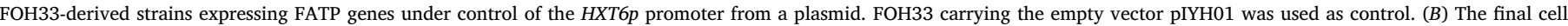

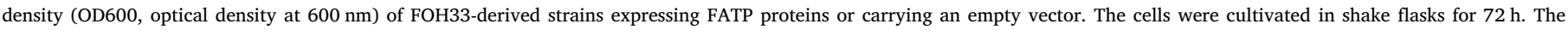
means \pm s.d. of biological triplicates are presented. Increased production of extracellular fatty alcohols $(+)$ or no effect $(-)$ compared to control strain is indicated according to $(A)$.

six new FATP chimeras, FATP423, FATP153, FATP126, FATP156, FATP426 and FATP453 (Fig. 3A). Each new FATP chimera still contained the well-conserved motifs from either FATP1 or FATP4.
These new FATPs were expressed in the FOH33 strain. The final biomass obtained from all of the strains expressing the new FATPs was much lower compared to the FATP1 and FATP4 expressing strains
A

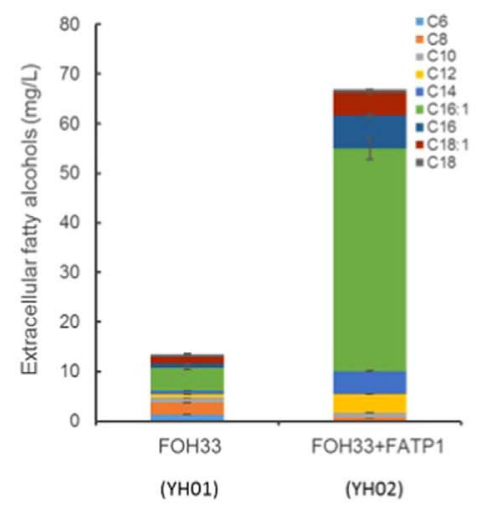

D

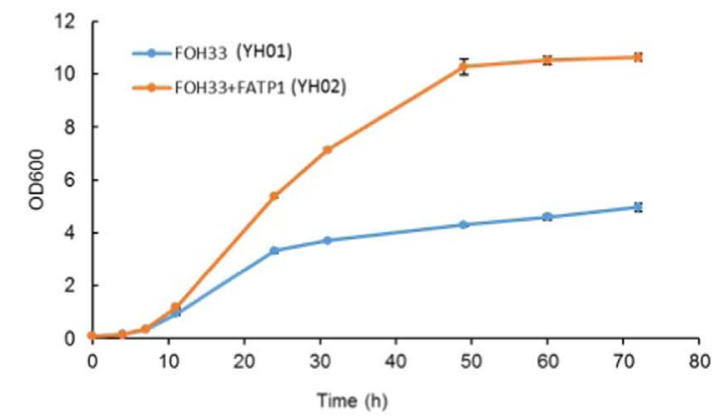

B

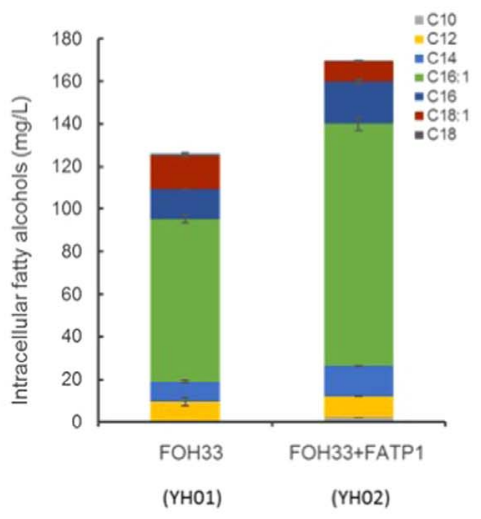

$\mathrm{E}$

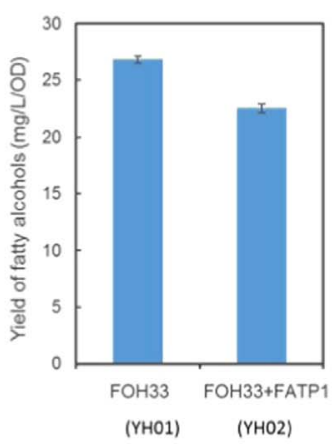

C
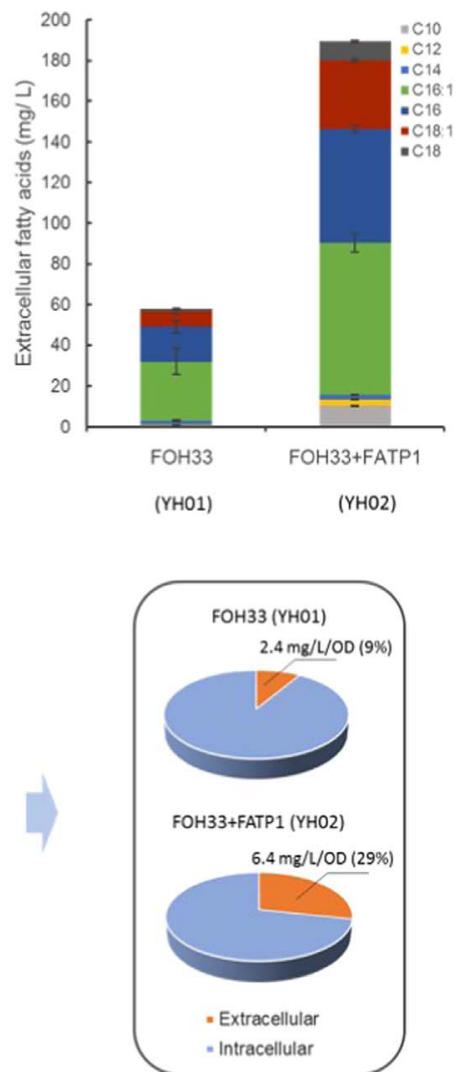

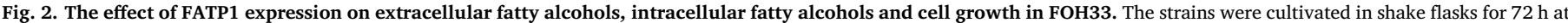

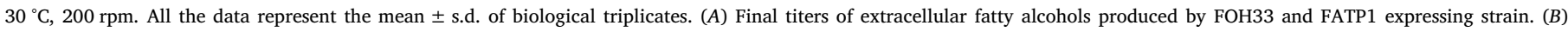

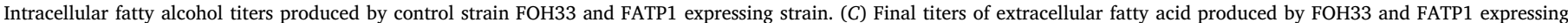

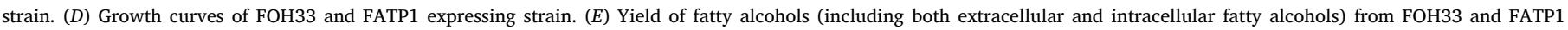
expressing strain, and the distribution of extracellular and intracellular fatty alcohols for each strain. 
A

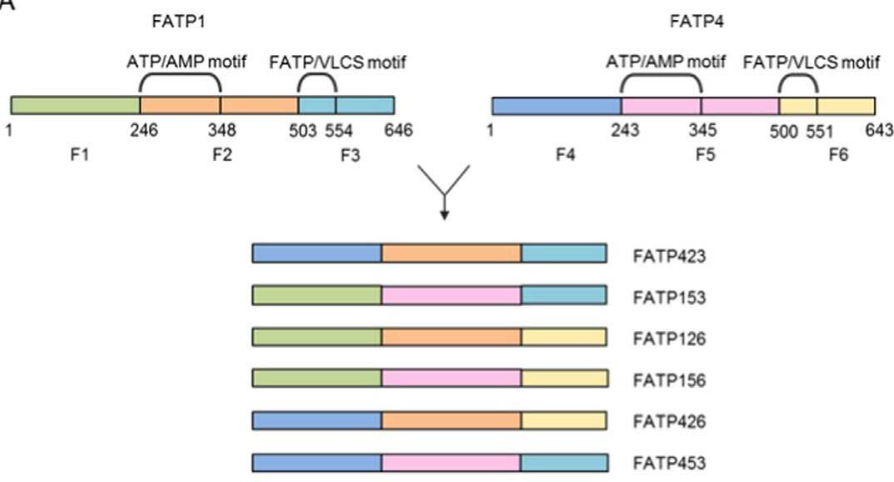

B

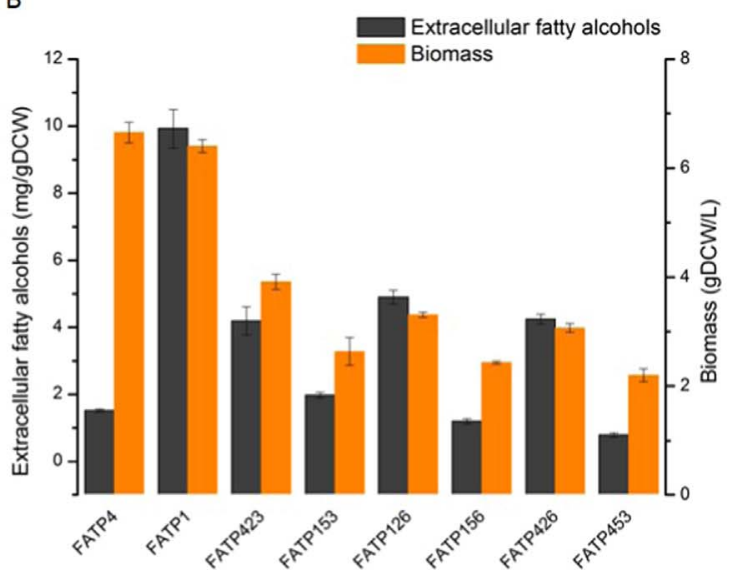

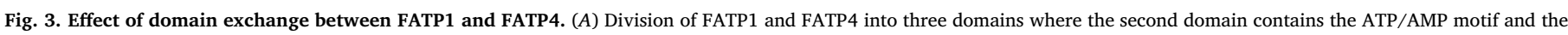

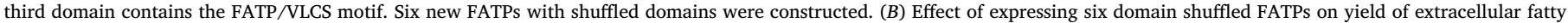
alcohols and the final biomass of each strain. The strains were cultivated in shake flasks for $72 \mathrm{~h}$ at $30^{\circ} \mathrm{C}, 200 \mathrm{rpm}$. All data represent the mean \pm s.d. of biological triplicates.

(Fig. 3B). These results suggest that the fatty acyl-CoA activity in the new isoforms may be affected after the domain exchange. However, these new isoforms showed different efficiencies in fatty alcohol transport. Based on the results, strains with FATP423, FATP126 and FATP426 showed an increased yield of extracellular fatty alcohols, while FATP153, FATP156 and FATP453 did not (Fig. 3B). The yield of extracellular fatty alcohols from strains containing FATP423, FATP126 and FATP426 was 3.9, 4.9 and $4.1 \mathrm{mg} / \mathrm{g}$ DCW, respectively, a more than 2-fold increase compared to the strain with FATP4. However, none of the new isoforms benefitted fatty alcohols secretion as efficiently as FATP1 did. All these three FATP chimeras shared the F2 domain containing the ATP/AMP motif from FATP1, which showed that the exchange of the second part of FATP4 alone (FATP426) was able to facilitate fatty alcohol export. This demonstrated that the F2 domain might represent the domain with the most important contribution to the fatty alcohol export activity.

\section{Discussion}

Two major hindrances of successful industrial fatty alcohol production using microbes are the low viability of cells and the complicated extraction processes of intracellular fatty alcohols. High amounts of fatty alcohols cause an inhibition of cell growth, which results in a limitation of fatty alcohol production. In order to improve the tolerance of cells towards fatty alcohols, we attempted to identify a transporter able to functionally export fatty alcohols from the yeast $S$. cerevisiae. As no fatty alcohol transporters have previously been identified in yeast or other organisms, there was limited information on potential transporter candidates available. Generally, transporters recognize substrates based on their structure. We assumed that certain fatty acid transporters might be able to transport fatty alcohols as well because of their similar structures. Therefore, fatty acid and wax transporters reported previously were evaluated here.

We found that FATP1 expression in yeast promoted the export of fatty alcohols (Fig. 1). Interestingly, it was reported that FATP1 is a membrane-bound acyl-CoA synthetase specific to very long chain fatty acids, and involved in uptake of long chain fatty acids (Coe et al., 1999; Glatz et al., 2010). In addition to elevated secretion of fatty alcohols, FATP1 expression in yeast also resulted in a higher biomass yield and thereby an increased overall fatty alcohol production. In the host strain FOH33, the deficiency of Faa1 and Faa4, which contribute predominantly to acyl-CoA synthetase activity, as well as expression of FaCoAR, a bifunctional reductase converting acyl-CoA to fatty alcohols, might cause a scarcity of acyl-CoA essential for cell growth. Therefore, one of the reasons for the observed biomass increase might be the VLC-
FACS activity of FATP1, i.e. the activation of very long chain fatty acids to form acyl-CoA thus complementing the FAA1/4 deletions in this strain. However, as the free fatty acid yield did not decrease, as one would expect for a faa1/4 complementation (data not shown), the overall cell fitness probably benefitted rather from the fatty alcohol export mediated by FATP1 than from its FACS activity.

Since there is a high similarity between all of the human FATP isoforms, we introduced also the other isoforms into the high fatty alcohol production strain FOH33. Both FATP1 and FATP4 were beneficial for cell growth, however only the FATP1 expressing strain showed activity of fatty alcohol export, which indicated that the functions of FACS and transport might be separated. From a domain exchange study between FATP1 and FATP4, the domain containing the ATP/AMP motif from FATP1 (amino acid residues 247-503) seemed to be the domain that contributed most to fatty alcohol export. Previous studies revealed that the amino acid residues within the FATP/VLACS motif of the yeast FATP orthologue Fat1 showed specific functions for fatty acid uptake (DiRusso et al., 2008; Zou et al., 2002). This indicates potential differences in mechanisms of fatty alcohol export and fatty acid uptake. Furthermore, the results of amino acid substitutions in Fat1 in a previous study showed that fat $1^{\mathrm{D} 508 \mathrm{~A}}$ had very limit effects on fatty acid transport but significantly reduced the FACS activity in yeast (Zou et al., 2002). The motif Y/F-X-X-G-D is one of the well conserved signature sequence shared within acyl-CoA synthetases, and the Asp ${ }^{508}$ residue in this motif plays an important role in ATP binding via hydrogen bonds with 2' and/or 3'- hydroxyl group of the ribose moiety (Hisanaga et al., 2004; Kochan et al., 2009). Thus, two mutations $\left(\mathrm{Asp}^{492} \mathrm{Ala}\right.$ and $\mathrm{Asp}^{492} \mathrm{Glu}$ ) at the corresponding position in FATP1 were introduced and the resulting proteins were expressed in FOH33. Both mutants lost their function as fatty alcohol transporter, indicating that fatty alcohol export might be an ATP-dependent process (Fig. S4). This result was also consistent with the hypothesis that the domain of FATP1 including the ATP/AMP motif played a critical role in the fatty alcohol export process.

To the best of our knowledge, this is the first study to identify a fatty alcohol transporter. We significantly improved the fitness of a biofuel production strain as well as the overall production and secretion by transporter engineering. We also identified a protein domain of FATP1 involved in fatty alcohol export. Although additional structural studies and protein engineering approaches of FATP1 should be conducted to further increase fatty alcohol secretion and help to understand the transport mechanism, this study highlights the possibility that transporter engineering could be a promising strategy to enable the successful commercialization of biofuel production. 


\section{Conclusion}

In this study, we first identified a human transporter that enabled fatty alcohol export and a resulting increased cell fitness in yeast. Around $70 \mathrm{mg} / \mathrm{L}$ extracellular fatty alcohols were obtained, which was 4.5-fold higher than the final titer of the control strain, when FATP1 was expressed in yeast. In addition, a more than 2-fold increase in biomass was achieved in FATP1 expression strain compared to the control strain. Further efforts towards functional domain identification were made through domain exchange. The results demonstrated that the F2 domain might have most important contribution to fatty alcohol export activity. This work revealed a promising transporter engineering strategy to a successful commercialization of yeast cell factories producing fatty alcohols and potentially other biofuels.

\section{Acknowledgements}

We thank Yongjin Zhou for useful suggestions about fatty alcohol extraction and analysis methods. This work was funded by the Knut and Alice Wallenberg Foundation, the Novo Nordisk Foundation, and the Swedish Foundation for Strategic Research. In addition, this project has received funding from the European Union's Horizon 2020 Framework Programme for Research and Innovation - Grant Agreement No. 720824.

\section{Appendix A. Supporting information}

Supplementary data associated with this article can be found in the online version at http://dx.doi.org/10.1016/j.ymben.2017.11.008.

\section{References}

Alper, H., Moxley, J., Nevoigt, E., Fink, G.R., Stephanopoulos, G., 2006. Engineering yeast transcription machinery for improved ethanol tolerance and production. Science 314, 1565-1568.

Bird, D., Beisson, F., Brigham, A., Shin, J., Greer, S., Jetter, R., Kunst, L., Wu, X., Yephremov, A., Samuels, L., 2007. Characterization of Arabidopsis ABCG11/WBC11, an ATP binding cassette $(\mathrm{ABC})$ transporter that is required for cuticular lipid secretion. Plant J. 52, 485-498.

Black, P.N., Dirusso, C.C., Sherin, D., Maccoll, R., Knudsent, J., Weimar, J.D., 2000. Affinity Labeling Fatty Acyl-coa Synthetase with 9-p-azidophenoxy Nonanoic Acid and the Identification of the Fatty Acid-binding Site 275. Publ. JBC Pap. Press, pp. 38547-38553.

Black, P.N., Sandoval, A., Arias-Barrau, E., DiRusso, C.C., 2009. Targeting the fatty acid transport proteins (FATP) to understand the mechanisms linking fatty acid transport to metabolism. Immunol. Endocr. Metab. Agents Med. Chem. 9, 11-17.

Black, P.N., Zhang, Q., Weimar, J.D., Dirusso, C.C., 1997. Mutational analysis of a fatty acyl-coenzyme a synthetase signature motif identifies seven amino acid residues that modulate fatty acid substrate specificity. J. Biol. Chem. 272, 4896-4903.

Bonen, A., Campbell, S.E., Benton, C.R., Chabowski, A., Coort, S.L.M., Han, X.X., Koonen, D.P.Y., Glatz, J.F.C., Luiken, J.J.F.P., 2004. Regulation of fatty acid transport by fatty acid translocase/CD36. Proc. Nutr. Soc. 63, 245-249.

Chen, Y., Daviet, L., Schalk, M., Siewers, V., Nielsen, J., 2013. Establishing a platform cell factory through engineering of yeast acetyl-CoA metabolism. Metab. Eng. 15, 48-54.

Chiu, H.-C., Kovacs, A., Blanton, R.M., Han, X., Courtois, M., Weinheimer, C.J., Yamada, K.A., Brunet, S., Xu, H., Nerbonne, J.M., Welch, M.J., Fettig, N.M., Sharp, T.L., Sambandam, N., Olson, K.M., Ory, D.S., Schaffer, J.E., 2005. Transgenic expression of fatty acid transport protein 1 in the heart causes lipotoxic cardiomyopathy. Circ. Res. 96, 225-233.

Coe, N.R., Smith, A.J., Frohnert, B.I., Watkins, P.A., Bernlohr, D.A., 1999. The fatty acid transport protein (FATP1) is a very long chain acyl-CoA synthetase. J. Biol. Chem. 274, 36300-36304.

DiRusso, C.C., Darwis, D., Obermeyer, T., Black, P.N., 2008. Functional domains of the fatty acid transport proteins: studies using protein chimeras. Biochim. Biophys. Acta $1781,135-143$.

DiRusso, C.C., Li, H., Darwis, D., Watkins, P.A., Berger, J., Black, P.N., 2005. Comparative biochemical studies of the murine fatty acid transport proteins (FATP) expressed in yeast. J. Biol. Chem. 280, 16829-16837.

Doege, H., Baillie, R.A., Ortegon, A.M., Tsang, B., Wu, Q., Punreddy, S., Hirsch, D., Watson, N., Gimeno, R.E., Stahl, A., 2006. Targeted deletion of FATP5 reveals multiple functions in liver metabolism: alterations in hepatic lipid homeostasis. Gastroenterology 130, 1245-1258.

Doshi, R., Nguyen, T., Chang, G., 2013. Transporter-mediated biofuel secretion. Proc. Natl. Acad. Sci. USA 110, 7642-7647.

Dunlop, M.J., 2011. Engineering microbes for tolerance to next- generation biofuels. Biotechnol. Biofuels 4, 32.
Dunlop, M.J., Dossani, Z.Y., Szmidt, H.L., Chu, H.C., Lee, T.S., Keasling, J.D., Hadi, M.Z., Mukhopadhyay, A., 2011. Engineering microbial biofuel tolerance and export using efflux pumps. Mol. Syst. Biol. 7, 487.

Falcon, A., Doege, H., Fluitt, A., Tsang, B., Watson, N., Kay, M.A., Stahl, A., 2010. FATP2 is a hepatic fatty acid transporter and peroxisomal very long-chain acyl-CoA synthetase. AJP Endocrinol. Metab. 299, E384-E393.

Fisher, M.A., Boyarskiy, S., Yamada, M.R., Kong, N., Bauer, S., Tullman-Ercek, D., 2014. Enhancing tolerance to short-chain alcohols by engineering the Escherichia coli AcrB efflux pump to secrete the non-native substrate n-butanol. ASC Synth. Biol. 3, 30-40.

Fortman, J.L., Chhabra, S., Mukhopadhyay, A., Chou, H., Lee, T.S., Steen, E., Keasling, J.D., 2008. Biofuel alternatives to ethanol: pumping the microbial well. Trends Biotechnol. 26, 375-381.

Gibson, D.G., Young, L., Chuang, R.-Y., Venter, J.C., Hutchison, C.A., Smith, H.O., 2009. Enzymatic assembly of DNA molecules up to several hundred kilobases. Nat. Methods 6, 343-345.

Gimeno, R.E., Ortegon, A.M., Patel, S., Punreddy, S., Ge, P., Sun, Y., Lodish, H.F., Stahl, A., 2003. Characterization of a heart-specific fatty acid transport protein. J. Biol. Chem. 278, 16039-16044.

Glatz, J.F.C., Luiken, J.J.F.P., Bonen, A., 2010. Membrane fatty acid transporters as regulators of lipid metabolism: implications for metabolic disease. Physiol. Rev. 90, 367-417.

Halter, D., Sprong, H., Somerharju, P., Egmond, M.R., 2006. ABC lipid transporters: extruders, flippases, or flopless activators? FEBS Lett. 580, 1171-1177.

Hamacher, T., Boles, E., Gárdonyi, M., Hahn-Hägerdal, B., Becker, J., 2002. Characterization of the xylose-transporting properties of yeast hexose transporters and their influence on xylose utilization. Microbiology 148, 2783-2788.

Herrmann, T., Buchkremer, F., Gosch, I., Hall, A.M., Bernlohr, D.A., Stremmel, W., 2001a. Mouse fatty acid transport protein 4 (FATP4): characterization of the gene and functional assessment as a very long chain acyl-CoA synthetase. Gene 270, 31-40.

Herrmann, T., Buchkremer, F., Gosch, I., Hall, A.M., Bernlohr, D.A., Stremmel, W., 2001b. Mouse fatty acid transport protein 4 (FATP4): characterization of the gene and functional assessment as a very long chain acyl-CoA synthetase. Gene 270, 31-40.

Hirsch, D., Stahl, A., Lodish, H.F., 1998. A family of fatty acid transporters conserved from mycobacterium to man. Proc. Natl. Acad. Sci. USA 95, 8625-8629.

Hisanaga, Y., Ago, H., Nakagawa, N., Hamada, K., Ida, K., Yamamoto, M., Hori, T., Arii, Y., Sugahara, M., Kuramitsu, S., Yokoyama, S., Miyano, M., 2004. Structural basis of the substrate-specific two-step catalysis of long chain fatty acyl-CoA synthetase dimer. J. Biol. Chem. 279, 31717-31726.

Kerr, R.A., 2007. Global warming is changing the world. Science 316, 188-190.

Khoomrung, S., Chumnanpuen, P., Jansa-Ard, S., Ståhlman, M., Nookaew, I., Borén, J., Nielsen, J., 2013. Rapid quantification of yeast lipid using microwave-assisted total lipid extraction and HPLC-CAD. Anal. Chem. 85, 4912-4919.

Kieboom, J., Dennis, J.J., de Bont, J.A., Zylstra, G.J., 1998. Identification and molecular characterization of an efflux pump involved in Pseudomonas putida S12 solvent tolerance. J. Biol. Chem. 273, 85-91.

Kochan, G., Pilka, E.S., Von Delft, F., Oppermann, U., Yue, W.W., 2009. Structural snapshots for the conformation-dependent catalysis by human medium-chain acylcoenzyme A synthetase ACSM2A. J. Mol. Biol. 388, 997-1008.

Le Hir, R., Sorin, C., Chakraborti, D., Moritz, T., Schaller, H., Tellier, F., Robert, S., Morin, H., Bako, L., Bellini, C., 2013. ABCG9, ABCG11 and ABCG14 ABC transporters are required for vascular development in Arabidopsis. Plant J. 76, 811-824.

Li, H., Schmitz, O., Alper, H.S., 2016. Enabling glucose/xylose co-transport in yeast through the directed evolution of a sugar transporter. Appl. Microbiol. Biotechnol. 100, 10215-10223.

Li, N., Xu, C., Li-Beisson, Y., Philippar, K., 2016. Fatty acid and lipid transport in plant cells. Trends Plant Sci. 21, 145-158.

Martin, G., Nemoto, M., Gelman, L., Geffroy, S., Najib, J., Fruchart, J.-C., Roevens, P., de Martinville, B., Deeb, S., Auwerx, J., 2000. The human fatty acid transport protein-1 (SLC27A1; FATP-1) cDNA and gene: organization, chromosomal localization, and expression. genomics 66, 296-304.

McFarlane, H.E., Shin, J.J.H., Bird, D.A., Samuels, A.L., 2010. Arabidopsis ABCG transporters, which are required for export of diverse cuticular lipids, dimerize in different combinations. Plant Cell 22, 3066-3075.

Mihalik, S.J., Steinberg, S.J., Pei, Z., Park, J., Kim, D.G., Heinzer, A.K., Dacremont, G., Wanders, R.J.A., Cuebas, D.A., Smith, K.D., Watkins, P.A., 2002. Participation of two members of the very long-chain acyl-CoA synthetase family in bile acid synthesis and recycling. J. Biol. Chem. 277, 24771-24779.

Minty, J.J., Lesnefsky, A.A., Lin, F., Chen, Y., Zaroff, T.A., Veloso, A.B., Xie, B., Mcconnell, C.A., Ward, R.J., Schwartz, D.R., Rouillard, J.-M., Gao, Y., Gulari, E., Lin, X.N., 2011. Evolution combined with genomic study elucidates genetic bases of isobutanol tolerance in Escherichia coli. Microb. Cell Fact. 10, 18.

Nijland, J.G., Vos, E., Shin, H.Y., de Waal, P.P., Klaassen, P., Driessen, A.J.M., 2016. Improving pentose fermentation by preventing ubiquitination of hexose transporters in Saccharomyces cerevisiae. Biotechnol. Biofuels 9, 158.

Pei, Z., Fraisl, P., Berger, J., Jia, Z., Forss-Petter, S., Watkins, P.A., 2004. Mouse very longchain Acyl-CoA synthetase 3/fatty acid transport protein 3 catalyzes fatty acid activation but not fatty acid transport in MA-10 cells. J. Biol. Chem. 279, 54454-54462.

Rutter, C.D., Zhang, S., Rao, C.V., 2015. Engineering Yarrowia lipolytica for production of medium-chain fatty acids. Appl. Microbiol. Biotechnol. 99, 7359-7368.

Schaffer, J.E., Lodish, H.F., 1994. Expression cloning and characterization of a novel adipocyte long-chain fatty-acid transport protein. Cell 79, 427-436.

Schmuth, M., Ortegon, A.M., Man, M.-Q., Elias, P.M., Feingold, K.R., Stahl, A., 2005. Differential expression of fatty acid transport proteins in epidermis and skin appendages. J. Investig. Dermatol. 125, 1174-1181.

Shin, H.Y., Nijland, J.G., de Waal, P.P., Driessen, A.J.M., 2017. The amino-terminal tail of Hxt11 confers membrane stability to the Hxt2 sugar transporter and improves xylose 
fermentation in the presence of acetic acid. Biotechnol. Bioeng. 114, 1937-1945.

Stahl, A., 2001. Fatty acid transport proteins: a current view of a growing family. Trends Endocrinol. Metab. 12, 266-273.

Stephanopoulos, G., 2007. Challenges in engineering microbes for biofuels production. Science 315, 801-804 (2).

Tang, X., Chen, W.N., 2015. Enhanced production of fatty alcohols by engineering the TAGs synthesis pathway in Saccharomyces cerevisiae. Biotechnol. Bioeng. 112, 386-392.

Turner, W.J., Dunlop, M.J., 2015. Trade-offs in improving biofuel tolerance using combinations of efflux pumps. ACS Synth. Biol. 4, 1056-1063.

Van Helvoort, A., Smith, A.J., 1996. MDR1 p-glycoprotein is a lipid translocase of broad specificity, while MDR3 p-glycoprotein specifically translocates phosphatidylcholine. Cell 87, 507-517.

Verduyn, C., Postma, E., Scheffers, W.A., Van Dijken, J.P., 1992. Effect of benzoic acid on metabolic fluxes in yeasts: a continuous-culture study on the regulation of respiration and alcoholic fermentation. Yeast 8, 501-517.

Watkins, P.A., Pevsner, J., Steinberg, S.J., 1999. Human very long.chain acyl.CoA synthetase and two human homologs- initial characterization and relationship to fatty acid transport protein. Prostaglandins, Leukot. Essent. Fat. Acids 60, 323-328.

Xu, S., Jay, A., Brunaldi, K., Huang, N., Hamilton, J.A., 2013. CD36 Enhances fatty acid uptake by increasing the rate of intracellular esterification but not transport across the plasma membrane. Biochemistry 52, 7254-7261.

Youngquist, J.T., Schumacher, M.H., Rose, J.P., Raines, T.C., Politz, M.C., Copeland, M.F., Pfleger, B.F., 2013. Production of medium chain length fatty alcohols from glucose in Escherichia coli. Metab. Eng. 20, 177-186.

Zhou, Y.J., Buijs, N.A., Siewers, V., Nielsen, J., 2014. Fatty acid-derived biofuels and chemicals production in Saccharomyces cerevisiae. Front. Bioeng. Biotechnol. 2, 32

Zhou, Y.J., Buijs, N.A., Zhu, Z., Qin, J., Siewers, V., Nielsen, J., 2016. Production of fatty acid-derived oleochemicals and biofuels by synthetic yeast cell factories. Nat. Commun. 7, 11709.

Zou, Z., Dirusso, C.C., Ctrnacta, V., Black, P.N., 2002. Fatty acid transport in Saccharomyces cerevisiae: directed mutagenesis of FAT1 distinguishes the biochemical activities associated with Fat1p. J. Biol. Chem. 277, 31062-31071. 